\title{
A Review on Data-driven Predictive Maintenance Approach for Hydro Turbines/Generators
}

\author{
Shewei Wang ${ }^{1,2}$, Kesheng Wang*, ${ }^{*}$, Zhe $\mathrm{Li}^{1}$ \\ 1. Department of Production and Quality Engineering, \\ Norwegian University of Science and Technology, \\ Trondheim, Norway \\ kesheng.wang@ntnu.no* \\ 2. College of Information Science and Engineering \\ Henan University of Technology \\ Zhengzhou, China
}

\begin{abstract}
Hydroelectricity as a renewable energy to respond the increasing population and environment crisis is widely used in the world. With the Hydro Turbines/Generators (HTG) being more and more complicated, the maintenance play a more and more important role in the production management in the hydro power plant. Many researches had concentrated on the predictive maintenance for the HTG in recent years. From the perspective of data-driven, this paper reviews and summarizes the key techniques regarding data acquisition, data processing, data analysis and data mining for the predictive maintenance of HTG. Especially, it place emphasis on the data-driven models for the diagnostics and prognostics. Finally, the paper concludes the current practices and presents a future research work.
\end{abstract}

Keywords-Predictive maintenance; Hydro turbines generators; Data-driven model; Data mining

\section{INTRODUCTION}

With the acute growth of population, resource depletion, pollution and climate change, hydroelectricity become one kind of the clean, safe, substantial energy to fulfill the growing energy remand [1]. Hydroelectricity has been one of the most widely used renewable energies in the world. The global annual hydropower production has passed $3000 \mathrm{TWH}$, which cover $86.31 \%$ of the global renewable energy production in 2008 [2]. Modern Hydro Turbines/Generators (HTG) is a large complicated and automatic electromechanical system. Any unanticipated shutdowns of its equipment will be catastrophic in the safe and in the cost. To ensure the HTG performing efficiently and reliably during their useful life, the maintenance is one of the most important activities in hydropower plants [3].

The current practice of maintenance applied to the HTG is mainly the preventive maintenance [4]. To reduce the probability of failure or the degradation of the functioning of an item, the preventive maintenance is carried out at predetermined intervals or according to prescribed criteria. However, the preventive maintenance cannot eliminate the occurrence of random catastrophic failure. In addition, the cost of frequent maintenance rises with the increasing demand of product in both quality and complexity. [5] indicated the maintenance cost for industrial firms in the USA has increased by $10-15 \%$ per year since 1979 . The maintenance even can cost up to $70 \%$ of the total operation costs, if it is not performed properly [6].

Predictive maintenance is a more efficient approach to HTG [4]. It takes an "execute at the right time" philosophy and carries out appropriate maintenance work for maximizing the service life of equipment without increasing the risk of failure in accordance with condition state and the Remaining Useful Life (RUL) of the equipment. There are two types of important tasks in predictive maintenance actions. Diagnostics deals with the fault detection, isolation and identification when it occurs. Prognostics deals with fault detection before it occurs [7].

The condition analysis and the predictive of the RUL are both based on the intelligent analysis of big data of equipment state and operation. The predictive maintenance is a datadriven maintenance strategy. The data-driven predictive maintenance model is shown in the Figure 1.

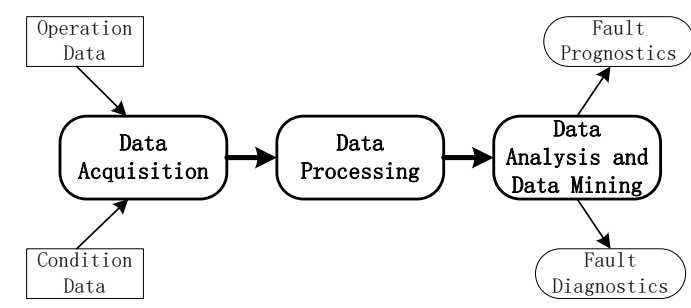

Figure 1. Data-driven predictive maintenance model

From the perspective of data processing, the predictive maintenance consists of three key steps: Data Acquisition, Data Processing, Data Analysis and Data Mining. Many researches had concentrated on the area of the diagnostics and prognostics for HTG from the recent literatures. However, the review or overview related to that topic is rare. This paper focuses on the review of the research on predictive maintenance approach for HTG and gives the systemic guidance to the further predictive maintenance research. The other part of the paper is organized as follows. In Section 2, we describe the sensors widely used in HTG. In Section 3, we review the general data processing methods for HTG. In Section 4, we review the models of data analysis and data mining for diagnostics and prognostics. Finally, in Section 5, we present the conclusion and the directions for the future work. 


\section{DATA ACQUISITION}

As the first step in predictive maintenance, data acquisition plays a critical role for the further data analysis and data mining, which is also vital to predictive maintenance for HTG. As shown in Figure 1, versatile condition data and operation data are required to collect to represent the physical asset state. The operation data write down what happened or what was done with the equipment, which are generally input and stored in the management information system. This paper will not cover the techniques of data acquisition and data processing for operation data. The condition data describe the physical state of the equipment. Due to the increasing complexity of equipment and increasing safety requirement, many kinds of sensors are installed on the HTG to monitor the main components of the system. Especially, in many HTG systems, the monitoring system has been integrated to the production system.

The condition data for HTG can be various types of physical and chemical quantities such as position, velocity, acceleration, vibration, torque, strain, temperature, acoustic, optical and infrared signals, microwaves, humidity, electric, electrolytic etc. The corresponding sensors also have a number of types: the mechanical, electric, electromechanical, optical, electrolytic, chemical, photochemical, field-effect and masssensitive and so on. Examples of different types of sensors equipped with HTG are shown in Table 1.

TABLE 1. SENSOR TYPE AND MEASUREMENT FOR HTG

\begin{tabular}{cc}
\hline Measurement/Component & Sensor Type \\
\hline $\begin{array}{c}\text { Temperature / Generator, } \\
\text { Bearing }\end{array}$ & Temperature sensor \\
$\begin{array}{c}\text { Vibration / Bearing, Stator } \\
\text { frame, Turbine cover, Draft } \\
\text { tube }\end{array}$ & Velocity sensor \\
Vibration / Shaft & Eddy current probe \\
Cavitation / Turbine, Inlet & Accelerometer sensor \\
guide vanes & Partial discharge probe \\
Partial discharge / Generator & Air gap sensor \\
Air gap / Generator & Eddy current probe \\
Position / Thrust bearing & Eddy current probe \\
Speed / Generator, Turbine, & Shaft
\end{tabular}

In summary, the most widely used sensors for HTG are temperature sensor, velocity sensor, accelerometer sensor, partial discharge sensor, air gap sensor and eddy current sensor. Among them, the accelerometer, the partial discharge, the air gap and the eddy current sensor are more applied to HTG than to other fields. This section places emphasis on the four types of sensors.

\section{A. Accelerometer}

An accelerometer generally measures the values of dynamic acceleration caused by moving of the object. The output of the accelerometer is either analog or digital. Accelerometer sensor is commonly used in vibration for condition monitoring [8]. In HTG, the cavitation damage caused by metal erosion can induce the vibration of turbine and guide vanes. Monitoring high frequency vibration by accelerometer allows for vanes position or flow parameter correction.

\section{B. Partial Discharge}

The partial discharge is a kind of small electrical sparks occurring in the insulation of HTG. In general, the more of this activity arise, the closer the winding of the generator is to failure. Partial discharge measurement is extremely important technique to diagnose the insulation degradation for HTG. A wide variety of sensors are used to test the partial discharge such as radio frequency, capacitor etc. [9] presented a partial discharge detection method, in which a resistance-temperature detector laid in the stator windings is used along with a continuous wavelet transform to improve the signal to noise ratio.

\section{Air Gap}

The air gap measures the space between the spinning rotor and the stationary stator in a hydro generator. Monitoring the air gap distance provides an indication of the rotor position and shape to detect problems such as vibration, magnetic imbalance, offset center, fretting, misalignment, and excessive wear. There are three types of air gap sensors: the contacting, the non-contacting, and non-invasive sensors. [10] discussed the air gap monitoring and fault diagnosis for a slow-speed salient-pole generator of hydropower unit, and carried out a case study on non-contacting sensors

\section{Eddy Current Probe}

Eddy current testing is one of electromagnetic testing methods making use of electromagnetic induction to detect and characterize surface and sub-surface flaws in conductive materials. The eddy current are generally applied to the HTG to monitor the thrust position, turbine speed, rotor eccentric, shaft vibration, and so on. [11] presented a hydro monitoring system based on ADAM modules, in which the eddy current sensors are used to monitor displacement and vibration.

\section{DATA PROCESSING}

The condition data collected from sensors are generally time-varying or spatially varying signal data. In the data processing, the condition data need to be further transformed and processed with the signal processing procedure. Typical signal processing procedure includes denoising, statistics analysis, time-frequency transformation etc. During the signal processing, the raw sensor data are cleaned and further simplified with dimension-reducing operation. Signal processing methods for HTG can be roughly divided into three types: the time domain, the frequency domain and the timefrequency domain.

\section{A. Time Domain Methods}

The time domain methods process condition signal data directly in waveform without transforming into another domain (e.g. frequency domain). Statistical methods are frequently applied to condition signals. Some statistical values are 
computed as indicators related to some process characteristics. For example, The Peak-to-valley, RMS, and Crest Factor describe the variation of the waveform signal from the perspectives of the absolute value, mean value and relative value respectively, which are given by

$$
\begin{aligned}
& \text { Peak }- \text { to }- \text { valley }=\max (x(n))-\min (x(n)) \\
& \text { RMS }=\sqrt{\frac{1}{N} \sum_{i=1}^{N}(x(i)-\bar{x})^{2}} \\
& \text { Crest Factor }=\frac{\text { Peak }- \text { to }- \text { valley }}{\text { RMS }}
\end{aligned}
$$

where $x(n), N$ and $\bar{x}$ denote the discrete signal values, the number of the discrete values, and the mean of the signal respectively. Many mechanical faults will increase the RMS of the vibration of certain components eventually. [12] investigated the RMS and Crest Factor of the gear mesh stiffness for the vibration-based fault detection and presented a new method for a propagating crack in the tooth root.

Time synchronous averaging (TSA) is another commonly used approach for time domain signal. TSA is an average of the raw signal over a number of evolutions which is given by

$$
T S A=\frac{1}{N} \sum_{n=0}^{N-1} s(t+n T), 0 \leq t \leq T_{x}
$$

where $\mathrm{s}(\mathrm{t})$ denotes the signal, $\mathrm{T}$ is the averaging period and $\mathrm{N}$ is the number of samples. TSA is applicable to many independent variable domains that demonstrate periodic signals which can be applicable to the spatial domain. TSA is often used as a preprocessor to enhance the signal to noise ratio of a periodic signal. In conjunction with conventional TRA, [13] presented a multiple-pulse individually rescaled-time synchronous averaging technique for fault detection of gears. The method was adopted as signal filtering technique to improve the signal to noise ratio for better feature extraction.

\section{B. Frequency Domain Methods}

The frequency domain methods deal with the signal data in frequency domain, in which the time based waveform data are transformed into frequency domain. The Fast Fourier Transform algorithm is a very popular method for frequency domain transforming. In the frequency domain, some frequency analysis approaches are carried to extracted valuable features. The commonly used approaches are: spectrum analysis, frequency filter, envelope analysis etc. Power spectrum, order spectrum and cepstrum are often used in fault diagnostics. Signal filtering is accomplished by applying different weights to different frequencies, e.g. highpass, lowpass, bandpass/stop filters.

\section{Time - Frequency Methods}

The time-frequency methods deal with signal data in both time and frequency domain. The non-stationary signal is common for the HTG whose distribution of energy over frequencies changes over time. The traditional spectral analysis is not adequate to handle the periodic or aperiodic changes. The general time-frequency analysis uses time-frequency distributions such as Short-time Fourier Transform, WignerVille distribution and so on [7]. Wavelet transform is another type of time-frequency method that can produce high frequency resolution at low frequencies and high time resolution at high frequencies by a time-scale representation of the signal. Wavelet transform has been widely applied to bearing fault detection, mechanical fault diagnosis and wind turbine transmission system fault diagnosis etc.

\section{MODELS FOR DiAgNOSTICS AND PROGNOSTICS}

In the data analysis and data mining step, some modelbased approaches are applied to diagnostics and prognostics. The models widely used can be generally divided into three types: the physical models of the system, the stochastic models and the data-mining models. Many models, especially the stochastic models and the data-mining models are based on the statistics and analysis of the condition data of the equipment. This section will focus on the advantages, feasibility and limitations of each above models.

\section{A. Physical models}

Physical models are the descriptions of the predictive maintenance system using physical principles, mathematical formulas and system analysis methods. A physical model can analytically obtain the relationship between the system condition and the system performance. The physical models are often used to analyze the phenomenon and the characteristics of the faults of the HTG to obtain the principles of the faults. [14] presented a dynamic model for radial vibration of hydro turbine generator units to assist the machine health monitoring. A finite element (FE) rotor dynamic model of radial vibration taking account of operating conditions is proposed to deal with the complex boundary conditions and exciting forces. $[15,16]$ studied the cavitation in hydro turbines using the theoretical model, experimental methods. The Rayleigh-Plesset equation is used to describe the formation of cavitation. The cavitation detection, diagnosis and reduction methods are summarized. [17, 18] used an electrical methods to diagnose the hydro generator insulation faults. The RF coupling method, the capacitive coupling method was used to the partial discharge analysis that indicates the insulation faults in the generator windings. Due to the complex structure regarding machinery, electric, control and other many auxiliary facilities, the single physical model is often effective on the analysis the certain specific fault of the HTG.

\section{B. Stochastic Models}

It is difficult to establish the precise physical models for the HTG diagnosis and prognosis in most time. Based on the statistical methods and probability distributions analysis, stochastic models are sometimes used to estimate the occurrence of the faults and predictive the remaining use life for the components or the equipment in the HTG. There are two types of stochastic models for the diagnosis and prognosis of HTG: statistical methods and probability analysis.

1) Statistical methods: Sometimes, the simple statistics values may be effective indicators to the failures of the components and the equipment. Based on the lots of historical operation records, the statistical methods are often used to analyze the failure and the lifetime of the components of hydroelectric generator. [19] computed the average periods 
between shutdowns for the bearing, generator and other main components and developed an empirical distribution functions to describe the probability of shutdowns in relation to the time of operation between hydroelectric generator shutdowns. The methods can act as a guide in the durability analysis of small hydro power plant. [20] studied the characteristics of the insulation systems for hydro generators using the statistical methods. The result show most defects occur in the electric system and are caused due to aging and electrical, thermal and mechanical load. [21] performed a statistical analysis on the discrete events in the hydroelectric generator. The result show the simple statistical analysis in discrete variables may reveal important operational characteristics and may raise questions with respect to the system overall operation performance that may be indicating conditions not acceptable in the future.

2) Probability analysis: The further probability analysis based on probability distributions and probability models will be used in the probability evaluation. [20, 22] applied the Weibull distribution to evaluate the statistical lifetime of hydro generators based on insulation system and the failure rate of the components. The bathtub curve of failure rate will guide the maintenance strategies of the hydroelectric generator. Based on the stochastic characteristics of the vibration signal of hydro turbine, the cloud transformation and cloud reasoning are applied to them when they are inputted to the neuro network to train [23]. To deal with the complex cause of the hydroelectric generator fault, probability analysis and system analysis are ombined to provide an effective fault diagnosis method. [24] developed an applicable quantitative method for identifying the critical components for HTG faults. In the model of fault tree, the leaf node has a given probability value, then the fault probability of other nodes can be computed along the derivation relation in the tree.

\section{Data-mining Models}

The mapping between the parameters or variables of components and performance or conditions of the equipment is complex in HTG, which play a vital role in predictive maintenance. However, the physical models and the stochastic models are often insufficient to describe the mapping successfully. The sensors and SCADA system are widely equipped in the HTG. The increasing accumulated monitor data support a new approach to discover the mapping hide in the data: data mining. The data mining is a computational process of discovering patterns in large data sets, which are based database system and many artificial intelligence methods: machine learning, computer intelligence and statistic. Due to its excellent ability in processing the big data and complex issue, the data mining has been increasingly applied to predictive maintenance for hydroelectric generator and have shown improved performance over conventional approaches. The selection of the specific data mining methods have to be carefully considered according to the sensory data characteristics to obtain the best performance. The following are some data mining methods applied to the predictive maintenance of HTG from the recent literatures.
1) Artificial Neuro network: Artificial Neural Network (ANN) is a computational model inspired by nervous system, which is widely used for classification, data fitting and prediction [25]. Since ANNs differ from traditional statistical techniques in their ability to learn nonlinear features of a time series successfully, they have been widely used in fault diagnosis and prognosis. [4] applied the ANN to the predictive maintenance for electrohydraulic servomechanism of governing system in the hydro power plant. The result can monitor, identify and diagnosis the dynamic performance of the system. Before the ANN analysis, the physical model analysis may be effective to reduce the input factors. A recurrent neural network $(\mathrm{RNN})$ is a type of artificial neural network that can exhibit dynamic temporal behavior and have a better forecasting performance. [5] applied RNNs to predict the life span of the gear. The model can trace the deterioration rate of the gearbox, which can provide an early alarm when the predicted condition of the gearbox falls to a predefined dangerous level. [26] analyzed the gearbox health condition and deduced the major factors are bearing temperature, cooling oil temperature and the difference of the thermal difference of the gearbox. Then the ANN model is applied to predict the three factors, and all of them are used to evaluate the health condition of the gearbox. Especially, if delicately designed, the ANN also can predict the remaining use life of equipment or components. [27] presented an ANN model to predictive the remaining use life of the bearing. The model takes the lifetime and multiple vibration values as input and the remaining life percentage as the output.

2) Composite neuro network model: Artificial neuro network model has obvious advantages in its simplicity, parallelism, non-linear, but it is insufficient in the aspects of input parameter and structure optimization. Some other methods will be combined to the neuro network model to improve its ability in the fault diagnosis and prognosis. Since the input data of the neuro network are limited in the numerical values, the fuzzy logic is combined and form a fuzzy neuro network to deal with the fuzzy value. [28] designed the member function and applied the neuro fuzzy network to predict the hydroelectric generator fault based on the stator temperature. To reduce the randomness of the vibration signal, [23] presented a new algorithm-cloud neural network based on cloud transformation. The cloud neural network model was applied to the hydroelectric generator vibration fault diagnosis. As the principal component analysis is effective on dimensionality reduction, it is merged into the neural network to improve its data processing ability [29]. The Genetic Algorithm is a global optimization algorithm and has no limitation on problem complexity. It can provide an effective method to determine the structure the neuro network. [30] presented a neural network model based on genetic algorithm for the fault diagnosis of hydroelectric generator. The model was applied to three kinds of hydroelectric generator faults: eccentric vortex band, unbalance, and misalignment. 
3) Support Vector Machine: Lots of training data play an important role to many neuro network methods, but the abnormal performing data are always rare to many vital components in HTG. The support vector machine (SVM) is an excellent classifying technique that can be used to fault diagnosis and fault prognosis. Support vector machine are supervised learning models with associated learning algorithms that analyze data and recognize patterns, used for classification and regression analysis. The key advantage of SVM is effective for lack of training samples and it is often used in the HTG. [31] applied the SVM to the vibration fault diagnosis for the HTG. In the SVM models, the amplitude energy on the $(0.4 \sim 0.5) x, 1 x, 2 x, 3 x,>3 x$ segment in the frequency spectrum are selected as input features. The sample data are used to compute the support vectors. Based on the support vectors, the fault type of the HTG can be predicted. The SVM can also be used to evaluate the remaining use life. [32] presented a remaining use life prediction model based on the SVM. In the model, the time domain condition data for bearing are used to create a classification by SVM hyper planes. The RUL is to be estimated and the degradation speed is evaluated by computing the minimal distance defined based on the degradation trajectories.

4) Other data mining techniques: A decision tree is a classifier that uses a tree-like graph model of decisions, in which each internal node represents a computation condition of an attribute, each branch represents the outcome of the computation and each leaf node represents a class label. Due to the simplicity and the classifying speed, the decision tree is widely used in fault diagnosis and prognosis. [21] applied the decision tree to analyze the performance of the HTG. The turbine water discharge and the upstream water level are taken as the condition attributes, and the generator power is taken as target attribute. The result will helpful for the operation and maintenance. [33] applied the decision tree to equipment availability analysis. The result can indicate the machine that is most responsible for the low equipment availability, which will be used to improve maintenance.

Association analysis is a method for discovering interesting relations between variables in large databases. The discovery of association rules are based on computing the frequent item set which is a set of attributes occurred frequently. The attribute in the frequent item set have more strong relation which can be described with association rules. Such analysis is useful in many decision-making processes, such as fault diagnosis, operation performance etc. [21] applied the association analysis to historical records for the hydroelectric generators alarm. The result show there are strong relation between certain some generators according to their alarm occurrence time, which need further analysis and maybe helpful to improve the operation.

\section{CONCLUSION AND FUTURE WORK}

Maintenance plays a vital role in the production management for the hydro power plant. This paper summarizes recent research and development in predictive maintenance for
HTG. The predictive of condition and performance of the equipment is based on the big data analysis of the condition monitoring. The data-driven model and technique is the core of the predictive maintenance. Diverse models, techniques and algorithms are reviewed with respect to the main steps of the predictive maintenance such as data acquisition, data processing, data analysis and data mining. From the literatures, the data mining techniques applied to the maintenance of the HTG are relatively less. Especially, the systemic data mining approach based on big data of predictive maintenance for HTG is relatively lack at present. With the increasing accumulation of the sensor data and the growing importance of the predictive maintenance for HTG, more and more researches will focus on that topic.

In our future work, a progressive data-driven approach for predictive maintenance of HTG will be presented. It will consist of six modules: (1) maintenance sensors and data acquisition, (2) maintenance data signal processing and feature extraction, (3) maintenance data intelligent analysis and data mining, (4) maintenance key performance indicator, (5) maintenance decision making and optimization, (6) maintenance management. All of the modules are integrated to provide a systemic solution for the predictive maintenance of HTG.

\section{ACKNOWLEDGMENT}

The authors wish to thank the Knowledge Discovery Lab of Department of Production and Quality Engineering of Norwegian University of Science and Technology for their support.

\section{REFERENCES}

[1] Bilgen, S., K. Kaygusuz, and A. Sari, Renewable energy for a clean and sustainable future. Energy Sources, 2004. 26(12): p. 1119-1129.

[2] Lejeune, A. and S. Hui, Hydropower: a multi benefit solution for renewable energy. Compr Renew Energy, 2012. 6: p. 15-47.

[3] Li, Z.-H., et al. Maintenance-oriented information digitalization of hydro turbine generator sets. in Power \& Energy Society General Meeting, 2009. PES'09. IEEE. 2009. IEEE.

[4] Jiang, W. Research on predictive maintenance for hydropower plant based on MAS and NN. in Pervasive Computing and Applications, 2008. ICPCA 2008. Third International Conference on. 2008. IEEE.

[5] Yam, R., et al., Intelligent predictive decision support system for condition-based maintenance. The International Journal of Advanced Manufacturing Technology, 2001. 17(5): p. 383-391.

[6] $\mathrm{Hu}, \mathrm{Q}$., et al. A novel hybrid intelligent maintenance system and its application. in Services Systems and Services Management, 2005. Proceedings of ICSSSM'05. 2005 International Conference on. 2005. IEEE.

[7] Jardine, A.K., D. Lin, and D. Banjevic, A review on machinery diagnostics and prognostics implementing condition-based maintenance. Mechanical systems and signal processing, 2006. 20(7): p. 1483-1510.

[8] Safizadeh, M. and S. Latifi, Using multi-sensor data fusion for vibration fault diagnosis of rolling element bearings by accelerometer and load cell. Information Fusion, 2014. 18: p. 1-8.

[9] Kanegami, M., S. Miyazaki, and K. Miyake, Partial Discharge Detection with High-Frequency Band through Resistance-Temperature Sensor of Hydropower Generator Stator Windings. Electrical Engineering in Japan, 2016. 195(4): p. 9-15. 
[10] Griscenko, M. and R. Elmanis-Helmanis, Eccentricity Of Slow-Speed Salient-Pole Generator: Analysis Based On Air Gap Spectrum. Latvian Journal of Physics and Technical Sciences, 2015. 52(1): p. 26-37.

[11] Zhang, W. and Z. Tongji, Development and application of hydropower hydro monitoring system. Procedia Engineering, 2011. 15: p. 807-811.

[12] Mohammed, O.D., M. Rantatalo, and J.-O. Aidanpää, Improving mesh stiffness calculation of cracked gears for the purpose of vibration-based fault analysis. Engineering Failure Analysis, 2013. 34: p. 235-251.

[13] Ahamed, N., Y. Pandya, and A. Parey, Spur gear tooth root crack detection using time synchronous averaging under fluctuating speed. Measurement, 2014. 52: p. 1-11.

[14] Xu, Y., Z. Li, and X. Lai, Dynamic model for hydro-turbine generator units based on a database method for guide bearings. Shock and Vibration, 2013. 20(3): p. 411-421.

[15] Escaler, X., et al., Detection of cavitation in hydraulic turbines. Mechanical systems and signal processing, 2006. 20(4): p. 983-1007.

[16] Kumar, P. and R. Saini, Study of cavitation in hydro turbines-A review. Renewable and Sustainable Energy Reviews, 2010. 14(1): p. 374-383.

[17] Lyles, J.F., T.E. Goodeve, and G.C. Stone, Using Diagnostic Technology for Identifying Generator Winding Maintenance Needs. Hydro Review, 1993. 12(4): p. 58-67.

[18] Tavner, P., Review of condition monitoring of rotating electrical machines. Electric Power Applications, IET, 2008. 2(4): p. 215-247.

[19] Drab, A., J. Riha, and M. Spano, Failures and durability analysis of a small hydro power plant.

[20] Sumereder, C., Statistical lifetime of hydro generators and failure analysis. Dielectrics and Electrical Insulation, IEEE Transactions on, 2008. 15(3): p. 678-685.

[21] Ohana, I., U. Bezerra, and J.P.A. Vieira, Data-mining experiments on a hydroelectric power plant. Generation, Transmission \& Distribution, IET, 2012. 6(5): p. 395-403.

[22] Pansrisu, S. and S. Premrudeepreechacharn. Preventive maintenance optimization for excitation system of bhumibol hydro power plant. in Energy and Sustainable Development: Issues and Strategies (ESD), 2010 Proceedings of the International Conference on. 2010. IEEE.
[23] Liwei, H. and L. Zongkun. Research of Cloud Neural Network Based on Cloud Transformation and its Application on Vibration Fault Diagnosis of Hydro-Turbine Generating Unit. in Power and Energy Engineering Conference, 2009. APPEEC 2009. Asia-Pacific. 2009. IEEE.

[24] Badoniya, R., R. Premi, and P. Patel, System Failure Analysis in Hydro Power Plant. International Journal on Emerging Technologies, 2014. 5(1): p. 54.

[25] Wang, K., Applied computational intelligence in intelligent manufacturing systems. 2005: Advanced Knowledge International Pty.

[26] Garcia, M.C., M.A. Sanz-Bobi, and J. del Pico, SIMAP: Intelligent System for Predictive Maintenance: Application to the health condition monitoring of a windturbine gearbox. Computers in Industry, 2006. 57(6): p. 552-568.

[27] Tian, Z., An artificial neural network method for remaining useful life prediction of equipment subject to condition monitoring. Journal of Intelligent Manufacturing, 2012. 23(2): p. 227-237.

[28] Xu, Y. and Z. Wang. On a fault detection system based on neuro-fuzzy fusion method. in Control and Decision Conference (CCDC), 2010 Chinese. 2010. IEEE.

[29] Ji, Q.-L., et al. Study of fault diagnosis of hydro-generator unit via GA nonlinear principal component analysis neural network and Bayesian neural networks. in Machine Learning and Cybernetics, 2006 International Conference on. 2006. IEEE.

[30] Qiaoling, J., Q. Weimin, and C. Weiyou. Fault Diagnosis of HydroGenerator Unit via GA-Nonlinear Principal Component Analysis Neural Network. in Control Conference, 2007. CCC 2007. Chinese. 2007. IEEE.

[31] Yang, C., J. Tao, and J. Yu. Fault diagnosis of a hydro turbine generating set based on support vector machine. in Intelligent Systems, 2009. GCIS'09. WRI Global Congress on. 2009. IEEE.

[32] Galar, D., et al. Remaining useful life estimation using time trajectory tracking and support vector machines. in Journal of Physics: Conference Series. 2012. IOP Publishing.

[33] Wang, K., Applying data mining to manufacturing: the nature and implications. Journal of Intelligent Manufacturing, 2007. 18(4): p. $487-$ 495. 